\title{
Equinococose policística na Amazônia oriental brasileira: atualização da casuística
}

\author{
Polycystic echinococcosis in the Eastern Brazilian Amazon: an update
}

\author{
Manoel do Carmo Pereira Soares ${ }^{1}$, Carlos Augusto Moreira-Silva ${ }^{1}$, Max Moreira Alves ${ }^{1}$, Heloisa \\ Marceliano Nunes ${ }^{1}$, Ivanete Abraçado do Amaral ${ }^{2}$, Lizomar de Jesus Maués Pereira Móia ${ }^{2}$, \\ Simone Regina Souza da Silva Conde ${ }^{2}$, Fernanda Barbosa Almeida ${ }^{3}$ \\ Rosângela Rodrigues-Silva ${ }^{3}$ e José Ângelo Barletta Crescente ${ }^{4}$
}

\begin{abstract}
RESUM0
Mediante critérios epidemiológicos, clínicos e laboratoriais, foi levantada a casuística de equinococose policística no período de 1962 a 2003, no âmbito da Amazônia oriental brasileira, incluindo casos inéditos e aqueles já publicados. Dessa forma, foram identificados 40 casos da doença no referido período, compreendendo casos procedentes dos estados do Pará e Amapá, Brasil. A amplitude das idades foi de 10 a 72 anos. Do total 47,5\% pertenciam ao sexo masculino. 0 fígado foi o órgão mais acometido (82,5\% dos casos). 0 Echinococcus vogeli (Rausch e Bernstein, 1972), apresentou-se como o principal agente etiológico envolvido. A partir do reconhecimento da importância e das implicações do manejo da equinococose para a região tropical, acredita-se que deverá ocorrer uma implementação do diagnóstico precoce, tratamento adequado e de um melhor registro da doença.
\end{abstract}

Palavras-chaves: Equinococose. Hidatidose. Echinococcus vogeli.

\begin{abstract}
By means of epidemiological and clinical-laboratorial approaches was consolidated an update of polycystic echinococcosis in the Eastern Brazilian Amazon, period from 1962 to 2003, including unpublished cases and those already published. In that way, they were identified 40 cases of the disease in referred period, understanding cases coming from the States of Pará and Amapá, Brazil. The width of the ages went from 10 to 72 years and 47,5\% belonged to the masculine sex. The liver was the attacked organ (82,5\% of the cases). The Echinococcus vogeli (Rausch and Bernstein, 1972), comes as the main agent involved. Starting from the recognition of the importance and of the implications of the handling of the echinococcosis for the tropical area, it is believed that should happen an improvement of the diagnosis, appropriate treatment and of a better registration of the disease.
\end{abstract}

Key-words: Echinococcosis. Hidatidosis. Echinococcus vogeli.

Quatro espécies do gênero Echinococcus (Rudolphi, 1801) e cujas larvas podem infectar 0 homem, têm sido descritas: E. granulosus (Batsch, 1786), causa hidatidose unilocular e se distribui em todo o mundo; E. multilocularis ( Leuckart, 1863) , causa a hidatidose alveolar e é encontrada em algumas regiões árticas e algumas áreas da Europa; E. oligarthrus (Diesing, 1863), excepcionalmente pode produzir doença policística no homem e E. vogeli (Rausch e Bernstein, 1972), espécie de descrição mais recente, principal implicada na etiologia da hidatidose ( neotropical) policística que incide em regiões silvestres das Américas Central e do Sul ${ }^{456}$.

Na América tropical, o E vogeli apresenta como hospedeiro intermediário principal a Agouti paca (paca), além de outras poucas espécies de animais silvestres. Os hospedeiros definitivos são canídeos domésticos e silvestres. No ambiente silvestre destacase 0 Speothos venaticus (cachorro-do- mato vinagre) como 0 único hospedeiro natural conhecido desse Echinococcus. 0 homem infecta-se enquanto um hospedeiro intermediário acidental, tanto no ambiente silvestre como no doméstico ${ }^{4}$.

\footnotetext{
1. Instituto Evandro Chagas da Secretaria de Vigilância em Saúde do Ministério da Saúde, Belém, PA. 2. Hospital da Fundação Santa Casa de Misericórdia do Pará, Belém, PA. 3. Laboratório de Helmintos Parasitos de Vertebrados do Departamento de Helmintologia do Instituto Oswaldo Cruz da Fundação Oswaldo Cruz, Rio de Janeiro, RJ. 4. Hospital Universitário João de Barros da Universidade Federal do Pará, Belém, PA.

Endereço para correspondência: Dr. Manoel Soares. Seção de Hepatologia do Instituto Evandro Chagas. Av. Almirante Barroso 492, Marco, 66090-000 Belém, PA,

Telefax: 91 211-4418.

e-mail: manoelsoares@iec.pa.gov.br
} 
A mais recente revisão brasileira de equinococose policística neotropical publicada em 1996 por D'Alessandro et al ${ }^{6}$,aponta 20 casos para o Brasil, sete casos para a Amazônia ocidental, procedentes do Estado do Acre e apenas seis casos para a Amazônia oriental, sendo, destes, cinco procedentes do Estado do Pará e um do Amapá.

Este trabalho tem por objetivo resgatar e atualizar casuística dessa parasitose, de elevada letalidade e certamente subdiagnosticada, na Amazônia oriental brasileira.

\section{MATERIAL E MÉTODOS}

A casuística apresentada nesta atualização foi levantada no período de 1962 a 2003, no âmbito da Amazônia oriental brasileira, compreendendo os Estados do Pará e Amapá. Conforme consistência do diagnóstico epidemiológico, clínico, sorológico, de imagem (ultra-sonografia, tomografia computadorizada e/ou ressonância magnética) e prova terapêutica, foram incluídos: a) casos de equinococose já publicados, com ou sem a definição do agente etiológico; b) casos publicados com revisão da etiologia; c) casos não publicados da doença. Nos casos ainda não publicados e naqueles em que, embora já publicados, foi possível recuperar as lâminas histológicas da lesão, o diagnóstico etiológico baseou-se nas características dos acúleos rostelares (Figura 1) do parasita ${ }^{6}$. Aspectos histológicos complementares como morfologia e espessura das membranas laminar e germinativa foram considerados conforme descrito por Rausch et $\mathrm{al}^{21}$. Como rotina os tecidos foram corados por hematoxilina e eosina. Quando cabiam dúvidas, foi realizada coloração com PAS para melhor evidenciar as membranas.

Tanto o material a fresco como aquele fixado e corado em lâmina, foram examinados, mensurados e fotografados contando com um microscópio Axiophoto Zeiss, tanto na microscopia óptica convencional como com o concurso da microscopia óptica polarizada. Adaptado do resumo preconizado por D'Alessandro et al ${ }^{7}$, reproduz-se à seguir os critérios adotados para 0 presente estudo e referentes aos grandes e pequenos acúleos dos Echinococcus de importância nos trópicos (Tabela 1).

Para diagnóstico sorológico do gênero Echinococcus, foi empregado teste tipo immunoblot com antígenos oriundos do E. granulosus, conforme Romani ${ }^{22}$ e Ayadi et $\mathrm{al}^{2}$.

Tabela 1- Morfometria de grandes e pequenos acúleos de acordo com os critérios de Rausch et a ${ }^{21^{2 *}}$.

\begin{tabular}{|c|c|c|c|c|c|c|}
\hline & \multicolumn{3}{|c|}{ Grandes acúleos } & \multicolumn{3}{|c|}{ Pequenos acúleos } \\
\hline & $\begin{array}{l}\text { Tamanho } \\
\text { médio ( } \mu \mathrm{m})\end{array}$ & $\begin{array}{c}\text { Amplitude } \\
(\mu \mathrm{m})\end{array}$ & $\begin{array}{l}\text { Cabo/ } \\
\text { Lâmina }\end{array}$ & $\begin{array}{l}\text { Tamanho } \\
\text { médio }(\mu \mathrm{m})\end{array}$ & $\begin{array}{c}\text { Amplitude } \\
(\mu \mathrm{m})\end{array}$ & $\begin{array}{l}\text { Cabo/ } \\
\text { Lâmina }\end{array}$ \\
\hline E. vogeli & 42 & $38-46$ & $35 / 65$ & 33 & $30-37$ & $41 / 59$ \\
\hline E. oligarthrus & 32 & 29-38 & $43 / 57$ & 26 & $23-30$ & $43 / 57$ \\
\hline E. granulosus & 23 & $20-25$ & - & 19 & $15-21$ & • \\
\hline
\end{tabular}

\section{RESULTAD0S}

Foram levantados 40 casos para 0 período estudado, 39 $(97,5 \%)$ procedentes do Estado do Pará ( em um desses casos não foi possível obter a procedência precisa da localidade de origem), um caso (2,5\%) procedente do Estado do Amapá 36912131516171819232425283031 . A amplitude das idades dos pacientes foi de 10 a 72 anos com média de 41,73 e mediana de 45 anos. No que se refere ao sexo, 47,5\% pertenciam ao sexo masculino. Quanto ao local da lesão cística, 0 fígado foi 0 órgão mais comprometido, com 33 (82,5\%) casos. Outros órgãos afetados foram pulmão, intestino, coração, estômago, baço além de lesões em mesentério e peritônio. Dos casos procedentes do Pará, em 23 (59\%) houve 0 registro da etiologia, sendo que, destes, em 14 (60,9\%) 0 diagnóstico foi de Echinococcus sp, nove $(39,1 \%)$ teve como etiologia o E. vogeli. Em dois casos, originalmente com referência de etiologia para E. granulosus, foi possível a revisão da lâmina de um deles, redefinindo como agente etiológico 0 E. vogeli. 0 outro caso, devido a critérios clínicoepidemiológicos, e por persistirem dúvidas, diagnosticou-se como Echinococcus sp. Dois casos, originalmente diagnosticados como Echinococcus sp, foram revistos e confirmados como E vogeli. Ao caso procedente do Amapá foi atribuída a etiologia de E. vogeli ${ }^{17}$. 0 diagnóstico associando a microscopia óptica convencional e microscopia óptica de polarização, mostrou-se adequado na detecção de acúleos (Figuras 2, 3 e 4), protoescóleces ( Figura 4) e membranas císticas (Figura 5).

Na Tabela 2, estão discriminados os casos levantados e as principais características consoantes aos critérios padronizados

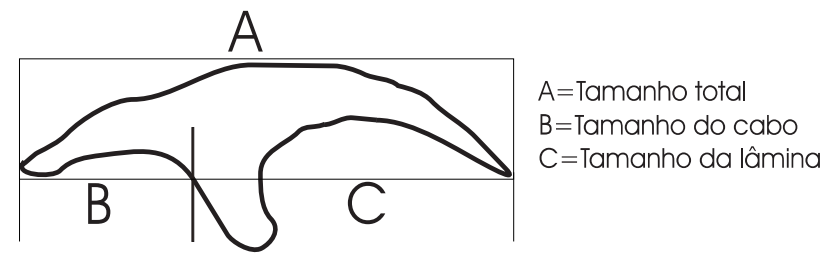

Figura 1 - Representação da medida dos acúleos de Echinococcus obedecendo ao critério proposto por Rausch et $\mathrm{al}^{21}$.

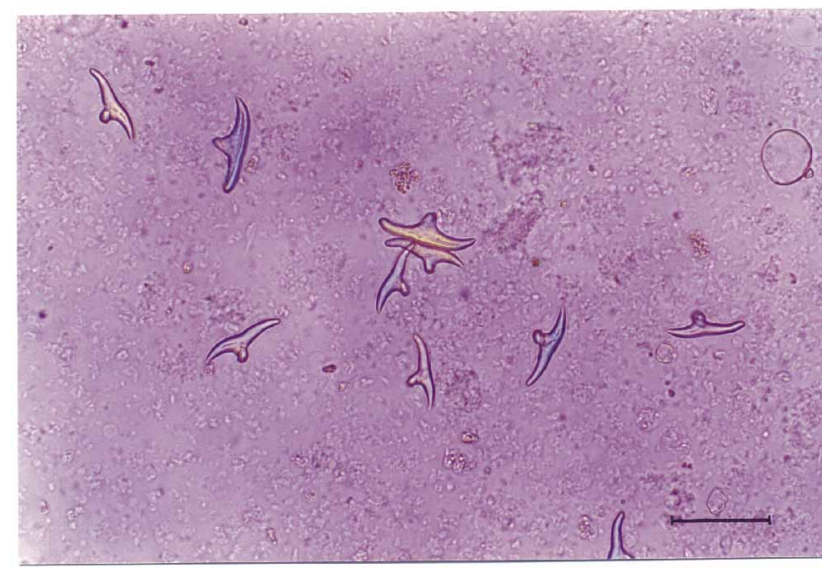

Figura 2 - Identificação de acúleos de E. vogeli por meio de microscopia óptica de polarização (material de líquido cístico) barra $=40 \mu \mathrm{m}$. 


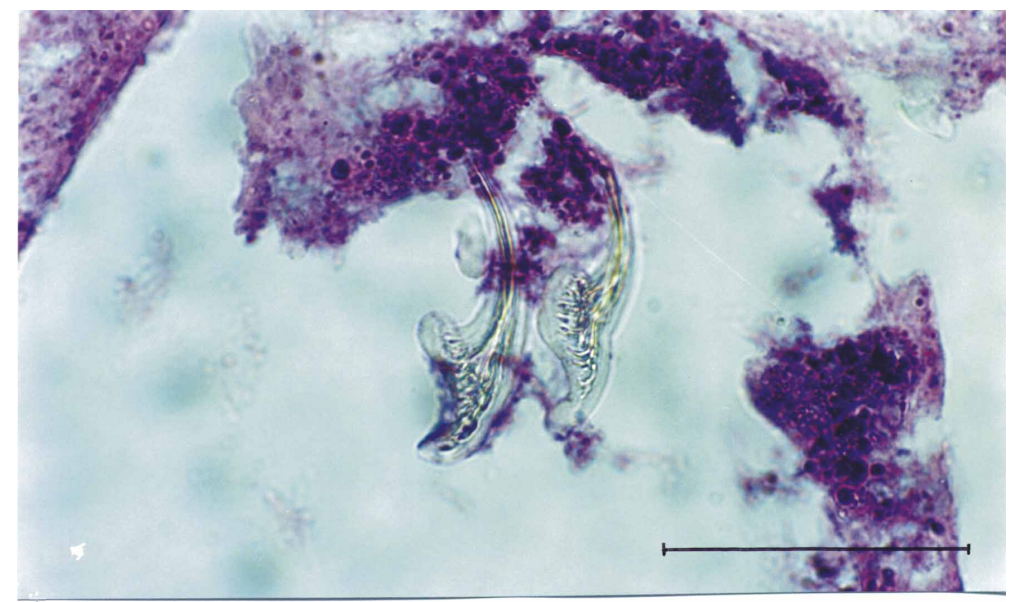

Figura 3 - Presença de dois acúleos típicos de Echinococcus vogeli, um maior (à esquerda) e outro menor (à direita). Lesão de linfonodo mesentérico. HematoxilinaEosina (barra $=40 \mu \mathrm{m})$.
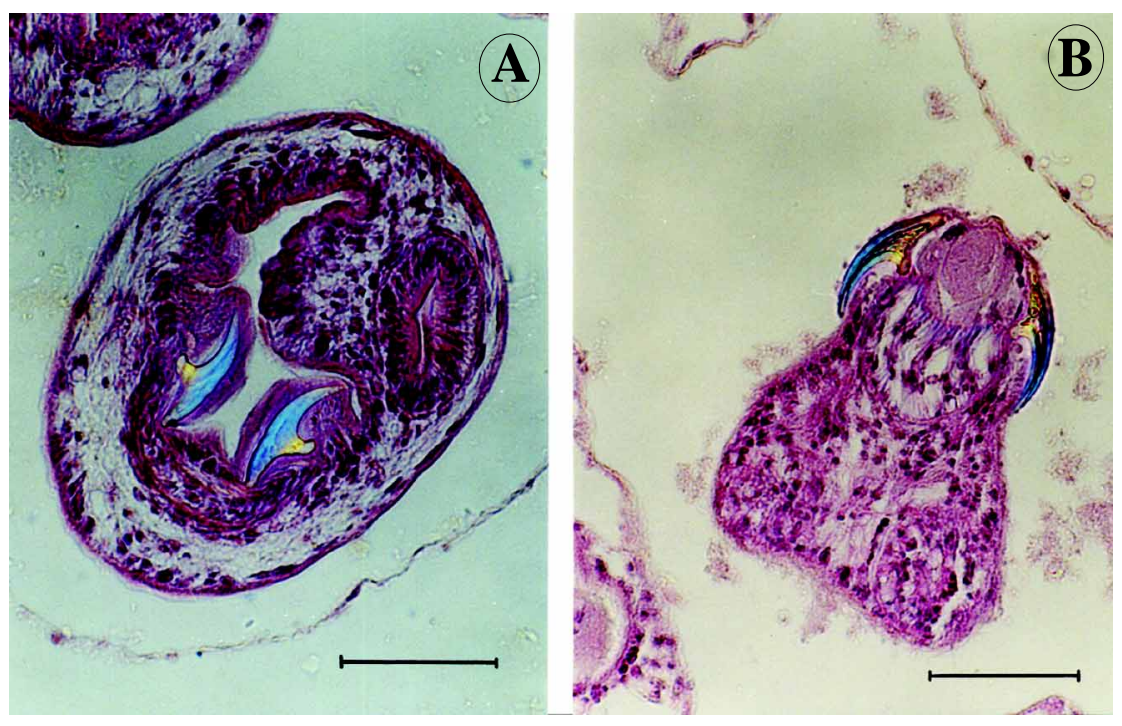

Figura 4 - Identificacão de protoescóleces e acúleos invaginados (A) e desinvaginados (B) de Echinococcus vogeli por meio de microscopia óptica de polarização. Hematoxilina-Eosina $($ barra $=40 \mu \mathrm{m})$.

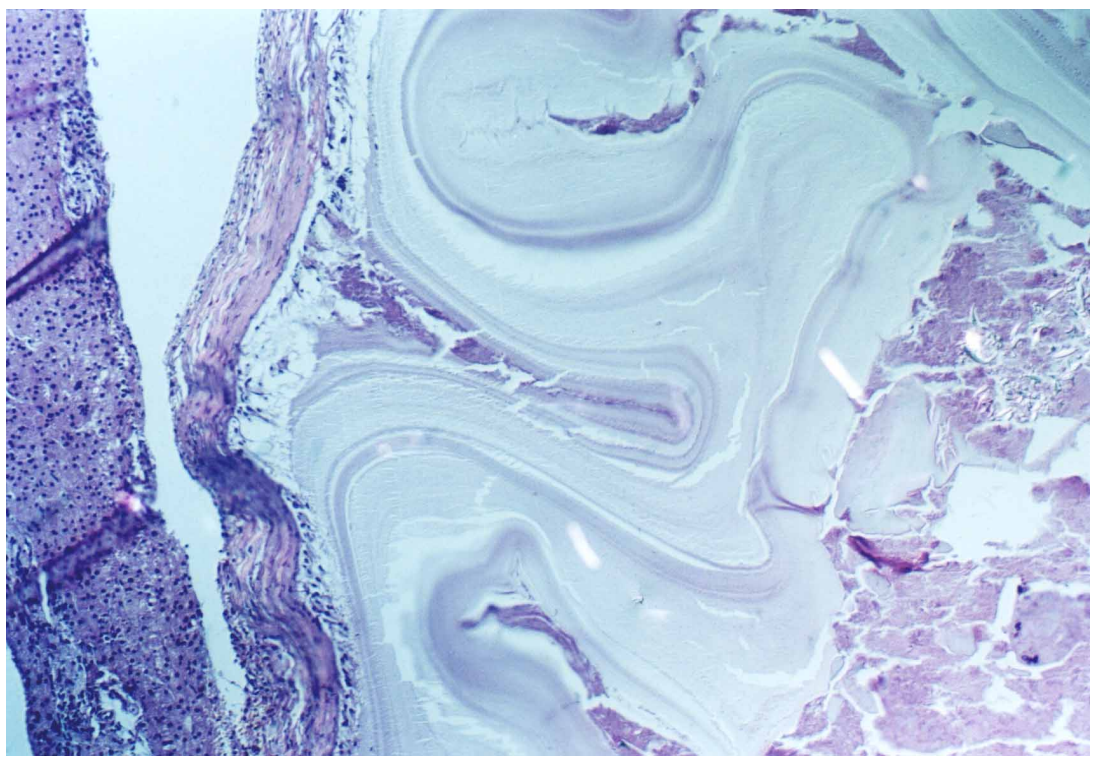

Figura 5 - Detalhe da membrana laminar multiplicada em direção ao interior do cisto (aspecto cerebróide), típico de Echinococcus vogeli. Lesão de fígado. Hematoxilina-Eosina. 
Tabela 2 - Casos humanos compatíveis com equinococose policística procedentes da Amazônia oriental brasileira. Período 1962 a 2003.

\begin{tabular}{|c|c|c|c|c|c|c|c|c|c|}
\hline Caso & $\begin{array}{l}\text { Município } \\
\text { (Estado) }\end{array}$ & $\begin{array}{l}\text { Idade } \\
\text { (anos) }\end{array}$ & Sexo & $\begin{array}{c}\text { Clínica/imagenologia/ } \\
\text { sorologia }\end{array}$ & $\begin{array}{l}\text { Local } \\
\text { do } \\
\text { cisto }\end{array}$ & Etiologia & Conduta invasiva & $\begin{array}{l}\text { Terapêutica / } \\
\text { evolução }\end{array}$ & Ref. \\
\hline 1 & $\begin{array}{l}\text { Curralinho/ } \\
\text { Ilha de Marajó } \\
\text { (PA) }\end{array}$ & 45 & $\mathrm{~F}$ & Desconhecidos & Fígado & E. $v$ & Biópsia & Desconhecida & 6 \\
\hline 2 & $\begin{array}{l}\text { Macapá } \\
(\mathrm{AP})\end{array}$ & 67 & $M$ & Doença não descrita & $\begin{array}{l}\text { Fígado e } \\
\text { Cólon }\end{array}$ & E. $v$ & Excisão Cirúrgica & Desconhecida & 6 \\
\hline 3 & $\begin{array}{l}\text { Moju } \\
(\mathrm{PA})\end{array}$ & 51 & $\mathrm{~F}$ & Desconhecidos & $\begin{array}{l}\text { Fígado e } \\
\text { Omento }\end{array}$ & - & Cirurgia & Desconhecida & 6 \\
\hline 4 & $\begin{array}{l}\text { Paragominas } \\
\quad \text { (PA) }\end{array}$ & 56 & M & $\begin{array}{l}\text { Dor e massa palpável no HD, } \\
\text { distensão abdominal, caquexia, } \\
\text { fístula de parede abdominal. }\end{array}$ & Fígado & E. $v$ & $\begin{array}{l}\text { Biopsia, } \\
\text { Laparotomia }\end{array}$ & Óbito & 6,9 \\
\hline 5 & (PA) & 43 & $\mathrm{~F}$ & $\begin{array}{c}\text { Perda ponderal, massa } \\
\text { abdominal, icterícia; } \\
\text { Calcificação linear (Raio-x). }\end{array}$ & Fígado & - & Cirurgia & Óbito & 19 \\
\hline 6 & $\begin{array}{c}\text { Campo Alegre/ } \\
\text { Santana do Araguaia } \\
\text { (PA) }\end{array}$ & 12 & $\mathrm{~F}$ & $\begin{array}{l}\text { Massa infra-umbilical } \\
\text { Hepatoesplenomegalia. }\end{array}$ & Mesentério & - & Cirurgia & Desconhecida & 18 \\
\hline 7 & $\begin{array}{l}\text { Marituba } \\
(\mathrm{PA})\end{array}$ & 49 & $\mathrm{~F}$ & Tumoração Abdominal. & Intestino & E. sp. & Laparotomia & Desconhecida & 9 \\
\hline 8 & $\begin{array}{l}\text { Breves/ } \\
\text { Ilha de Marajó } \\
\text { (PA) }\end{array}$ & 23 & M & $\begin{array}{l}\text { Dor no } \mathrm{HD} \text {, hepatomegalia com } \\
\text { bordos irregulares. }\end{array}$ & $\begin{array}{l}\text { Fígado e } \\
\text { Mesentério }\end{array}$ & E. sp. & Laparotomia & Desconhecida & 9 \\
\hline 9 & $\begin{array}{l}\text { Moju } \\
(\mathrm{PA})\end{array}$ & 59 & $\mathrm{~F}$ & $\begin{array}{l}\text { Tumoração abdominal, fígado } \\
\text { palpável e rugoso. }\end{array}$ & $\begin{array}{l}\text { Fígado, } \\
\text { Estômago, } \\
\text { Intestino e } \\
\text { peritônio. }\end{array}$ & E. sp. & Laparotomia & Desconhecida & 9 \\
\hline 10 & $\begin{array}{l}\text { Anajás/ } \\
\text { Ilha de Marajó } \\
\text { (PA) }\end{array}$ & 46 & $\mathrm{~F}$ & $\begin{array}{l}\text { Hepatomegalia dolorosa } \\
\text { Endurecida e irregular. }\end{array}$ & Fígado & E. sp. & Biópsia & Desconhecida & 9 \\
\hline 11 & $\begin{array}{l}\text { Anajás/ } \\
\text { Ilha de Marajó } \\
\text { (PA) }\end{array}$ & 50 & $\mathrm{~F}$ & $\begin{array}{c}\text { Massa endurecida em epigástrio } \\
\text { e icterícia. }\end{array}$ & Omento & E. sp. & Laparotomia & Desconhecida & 9 \\
\hline 12 & $\begin{array}{l}\text { Vila Nazaré/ } \\
\text { Dom Eliseu } \\
\text { (PA) }\end{array}$ & 10 & $\mathrm{~F}$ & Hepatoesplenomegalia. & Fígado & E. sp. & Biópsia & Desconhecida & 9 \\
\hline 13 & $\begin{array}{l}\text { Anajás/ } \\
\text { Ilha de Marajó } \\
\text { (PA) }\end{array}$ & 35 & $\mathrm{~F}$ & $\begin{array}{l}\text { Hepatomegalia dolorosa e } \\
\text { perda ponderal. }\end{array}$ & Fígado & E.sp. & Biópsia & $\begin{array}{c}\text { Mebendazol }(600 \\
\text { mg/dia }) \\
\text { Desconhecida }\end{array}$ & 9 \\
\hline 14 & $\begin{array}{l}\text { Cametá } \\
(\mathrm{PA})\end{array}$ & 45 & M & $\begin{array}{l}\text { Dor no HD e epigástrio, perda } \\
\text { ponderal, febre e icterícia. }\end{array}$ & Mesentério & E. v. & Cirurgia & $\begin{array}{l}\text { Albendazol }(600 \\
\text { mg/dia); } \\
\text { Desconhecida }\end{array}$ & 9,10 \\
\hline 15 & $\begin{array}{l}\text { Muanál } \\
\text { Ilha de Marajó } \\
\text { (PA) }\end{array}$ & - & M & Anasarca e cardiomegalia. & $\begin{array}{l}\text { Pulmão e } \\
\text { coração }\end{array}$ & E. sp. & Necropsia & óbito & 9 \\
\hline 16 & $\begin{array}{l}\text { Aldeia Kaiapó/ } \\
\text { Xingu } \\
\text { (PA) }\end{array}$ & - & M & $\begin{array}{l}\text { Edema agudo de pulmão, } \\
\text { septicemia. }\end{array}$ & Pulmão & E. sp. & Necropsia & Óbito & 9 \\
\hline 17 & $\begin{array}{l}\text { Afuál } \\
\text { Ilha de Marajó } \\
\text { (PA) }\end{array}$ & 32 & M & $\begin{array}{l}\text { Dor no HD, febre, tumoração } \\
\text { palpável no abdome e mal } \\
\text { estado geral. }\end{array}$ & $\begin{array}{l}\text { Fígado e } \\
\text { Mesentério }\end{array}$ & E. sp. & Laparotomia & Desconhecida & 9,17 \\
\hline 18 & $\begin{array}{l}\text { Curralinho/ } \\
\text { Ilha de Marajó } \\
\text { (PA) }\end{array}$ & 72 & M & $\begin{array}{l}\text { Hepatomegalia, icterícia e } \\
\text { tumoraçôes no HD. }\end{array}$ & Fígado & E. sp. & Biopsia & $\begin{array}{c}\text { Mebemdazol (400 } \\
\text { mg/dia) } \\
\text { Desconhecida }\end{array}$ & 9,16 \\
\hline 19 & $\begin{array}{l}\text { Serra Norte/ } \\
\text { Marabá } \\
\text { (PA) }\end{array}$ & 51 & M & $\begin{array}{l}\text { Dor no HD, colelitíase, dor } \\
\text { abdominal. }\end{array}$ & Fígado & E. sp. & Cirurgia & Desconhecida & 1,9 \\
\hline 20 & $\begin{array}{c}\text { Breves/ } \\
\text { Ilha de Marajó } \\
(\mathrm{PA})\end{array}$ & 33 & M & $\begin{array}{l}\text { Dor no HD, perda de peso, } \\
\text { nodulações no fígado e úlcera } \\
\text { em perna esquerda. }\end{array}$ & Fígado & E.v.. & Laparotomia & Desconhecida & 10 \\
\hline
\end{tabular}


Tabela 2 - Continuação.

\begin{tabular}{|c|c|c|c|c|c|c|c|c|c|}
\hline 21 & $\begin{array}{c}\text { Breves/ } \\
\text { Ilha de Marajó } \\
(\mathrm{PA})\end{array}$ & 48 & M & $\begin{array}{l}\text { Dor no HD, febre, icterícia, } \\
\text { hepatoesplenomegalia. }\end{array}$ & Fígado & E. sp. & Biópsia & Desconhecida & 10 \\
\hline 22 & $\begin{array}{l}\text { Anajás/ } \\
\text { Ilha de Marajó } \\
(\mathrm{PA})\end{array}$ & 43 & $\mathrm{~F}$ & $\begin{array}{c}\text { Dor em epigástrio e HD, febre, } \\
\text { icterícia, colúria, acolia, } \\
\text { massa palpável em epigástrio e } \\
\text { HD. }\end{array}$ & Fígado & E. sp. & - & Desconhecida & 10 \\
\hline 23 & $\begin{array}{l}\text { Portel/ } \\
\text { Ilha de Marajó } \\
\text { (PA) }\end{array}$ & 45 & M & $\begin{array}{l}\text { Febre, diarréia, calafrio, } \\
\text { cefaléia, abdome doloroso, } \\
\text { hepatomegalia. }\end{array}$ & Fígado & E.v. & $\begin{array}{c}\text { Biópsia e } \\
\text { Laparotomia }\end{array}$ & Desconhecida & 10 \\
\hline 24 & $\begin{array}{l}\text { Aldeia Parakanã } \\
\text { Itupiranga } \\
\text { (PA) }\end{array}$ & 28 & F & $\begin{array}{l}\text { Massa palpável em HD, } \\
\text { hepatomegalia, icterícia; } \\
\text { Pancistose hepática com } \\
\text { calcificações (TCA); } \\
\text { Sorologia Positiva. }\end{array}$ & Fígado & - & Não & $\begin{array}{l}\text { Albendazol ( } 400 \\
\text { mg/dia); } \\
\text { Melhora do quadro } \\
\text { clínico e atualmente } \\
\text { está assintomática. }\end{array}$ & 15 \\
\hline 25 & $\begin{array}{l}\text { Ponta de Pedras/ } \\
\text { Ilha de Marajó } \\
\text { (PA) }\end{array}$ & 21 & M & $\begin{array}{c}\text { Febre, icterícia, ascite, perda de } \\
\text { ponderal, massa palpável em } \\
\text { HD; } \\
\text { Microscopia de escarro } \\
\text { (visualização dos acúleos); } \\
\text { Múltiplas formações císticas } \\
\text { hepáticas com calcificações } \\
\text { (TCA); } \\
\text { Sorologia Negativa. }\end{array}$ & Fígado & E. v. & Não & $\begin{array}{l}\text { Albendazol (400 } \\
\text { mg/dia); } \\
\text { Melhora do quadro } \\
\text { clínico e atualmente } \\
\text { está assintomático. }\end{array}$ & 28 \\
\hline 26 & $\begin{array}{l}\text { Oeiras do Pará } \\
\text { (PA) }\end{array}$ & 47 & $\mathrm{~F}$ & $\begin{array}{l}\text { Febre, dor abdominal, massa } \\
\text { palpável em HD, } \\
\text { Icterícia. } \\
\text { Sorologia Positiva. }\end{array}$ & Fígado & E. $v$. & Cirurgia & Desconhecida & 30 \\
\hline 27 & $\begin{array}{l}\text { Anajás/ } \\
\text { Ilha de Marajó } \\
(\mathrm{PA})\end{array}$ & 24 & $\mathrm{~F}$ & $\begin{array}{l}\text { Massa palpável em HD, icterícia } \\
\text { e perda de ponderal; } \\
\text { Cistos hepáticos apresentando } \\
\text { configuração em "cacho de } \\
\text { uva" com calcificações (TCA); } \\
\text { Sorologia Positiva. }\end{array}$ & Fígado & - & Não & $\begin{array}{l}\text { Albendazol (400 } \\
\text { mg/dia); } \\
\text { Melhora do quadro } \\
\text { clínico. }\end{array}$ & 30 \\
\hline 28 & $\begin{array}{l}\text { Altamira } \\
\text { (PA) }\end{array}$ & 41 & M & $\begin{array}{l}\text { Dispnéia, tosse produtiva com } \\
\text { expectoração purulenta, } \\
\text { adinamia, astenia e anasarca; } \\
\text { Múltiplos nódulos pulmonares } \\
\text { compatíveis com metástase } \\
\text { (TCA); } \\
\text { Sorologia Positiva. }\end{array}$ & $\begin{array}{l}\text { Fígado } \\
\text { e } \\
\text { Pulmão }\end{array}$ & E. $v$. & Necrópsia & óbito & 23 \\
\hline 29 & $\begin{array}{l}\text { Portel } \\
\text { (PA) }\end{array}$ & 17 & $\mathrm{~F}$ & $\begin{array}{l}\text { Massa abdominal palpável } \\
\text { dolorosa em mesogástrio; } \\
\text { Sorologia Positiva. }\end{array}$ & Mesentério & E. $v$. & Cirurgia & $\begin{array}{l}\text { Albendazol (400 } \\
\text { mg/dia); } \\
\text { Desconhecida }\end{array}$ & 03 \\
\hline 30 & $\begin{array}{l}\text { Portel } \\
\text { (PA) }\end{array}$ & 40 & $\mathrm{~F}$ & $\begin{array}{c}\text { Icterícia, febre, calafrio, } \\
\text { dispnéia, dor abdominal, massa } \\
\text { palpável em HD; } \\
\text { Múltiplas formações císticas no } \\
\text { fígado (TCA); } \\
\text { Sorologia Positiva. }\end{array}$ & Fígado & - & Não & $\begin{array}{c}\text { Albendazol } \\
\text { (400 mg/dia); } \\
\text { Melhora do quadro } \\
\text { clínico. }\end{array}$ & 23 \\
\hline 31 & $\begin{array}{l}\text { Breves / } \\
\text { Ilha de Marajó } \\
(\mathrm{PA})\end{array}$ & 49 & $\mathrm{~F}$ & $\begin{array}{l}\text { Dor abdominal irradiante para } \\
\text { HD, pirose, plenitude gástrica; } \\
\text { Múltiplas formações císticas } \\
\text { hepáticas apresentando } \\
\text { configuração em "cacho de } \\
\text { uva" com calcificações (TCA); } \\
\text { Sorologia Positiva. } \\
\text { Icterícia, hepatomegalia, } \\
\text { abdome distendido, edema de } \\
\text { MMII; }\end{array}$ & Fígado & - & Não & $\begin{array}{l}\text { Albendazol } \\
(400 \mathrm{mg} / \mathrm{dia}) ; \\
\text { Melhora do quadro } \\
\text { clínico, com } \\
\text { regressão das lesões } \\
\text { císticas. }\end{array}$ & 31 \\
\hline 32 & $\begin{array}{l}\text { Moju } \\
(\mathrm{PA})\end{array}$ & 54 & M & $\begin{array}{l}\text { Múltiplos cistos hepáticos com } \\
\text { calcificações laminares (TCA); } \\
\text { Sorologia Negativa. }\end{array}$ & Fígado & - & Cirurgia & Óbito & 23 \\
\hline 33 & $\begin{array}{l}\text { Breves/ } \\
\text { Itha de Marajó } \\
\text { (PA) }\end{array}$ & 67 & $\mathbf{M}$ & $\begin{array}{l}\text { Febre, dor em HD e epigástrio; } \\
\text { Pancistose hepática com } \\
\text { calcificaróos (TCA); } \\
\text { Sorologia Positiva. }\end{array}$ & Higado & - & Não & $\begin{array}{c}\text { Albendazol } \\
\text { (400 mg/dia); } \\
\text { Melhora do quadro }\end{array}$ & 23 \\
\hline 34 & $\begin{array}{l}\text { Curralinho / } \\
\text { llha de Marajó } \\
\text { (PA) }\end{array}$ & 35 & $M$ & $\begin{array}{l}\text { Figado com múltiplas } \\
\text { formações císticas de forma e } \\
\text { tamanhos variados e } \\
\text { calcificações laminares (TCA); } \\
\text { Sorologia Positiva. }\end{array}$ & Figado & - & Não & $\begin{array}{c}\text { clínico. } \\
\text { Albendazol ( } 400 \\
\text { mg/dia); } \\
\text { Melhora do quadro } \\
\text { clínico }\end{array}$ & 23 \\
\hline
\end{tabular}


Tabela 2 - Continuação.

\begin{tabular}{|c|c|c|c|c|c|c|c|c|c|}
\hline 35 & $\begin{array}{l}\text { Altumira } \\
\text { (PA) }\end{array}$ & 24 & M & $\begin{array}{l}\text { Dor abdominal, febre, massa } \\
\text { nodular abdominal em } \\
\text { mesogístrio; } \\
\text { Hepatoesplenomegalia e } \\
\text { múltiplas formaçóes císticas em } \\
\text { figado e baço (TCA); } \\
\text { Sorologia Positiva. }\end{array}$ & $\begin{array}{l}\text { Fígado, baço e } \\
\text { peritônio }\end{array}$ & - & Não & $\begin{array}{l}\text { Albendazol (800 } \\
\text { mg/dia); } \\
\text { Desconhecida }\end{array}$ & 12 \\
\hline 36 & $\begin{array}{l}\text { Curralinho / } \\
\text { Ilha de Marajó } \\
\text { (PA) }\end{array}$ & 29 & $F$ & 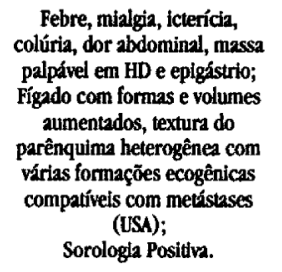 & Fígado & . & Não & Obito & 25 \\
\hline 37 & $\begin{array}{l}\text { Curuçá } \\
(\mathrm{PA})\end{array}$ & 54 & $\mathbf{F}$ & $\begin{array}{l}\text { Dor em Alanco e HD, antralgia, } \\
\text { mialgia, febre, calafrio, diarréia } \\
\text {, náuseas e vômitos; } \\
\text { Múltiplos cistos no figado com } \\
\text { calcificaçōes puntiformes no } \\
\text { lobo direito (TCA); } \\
\text { Sorologia Positiva. }\end{array}$ & Fígado & . & Não & Óbito & 25 \\
\hline 38 & $\begin{array}{l}\text { Braganf̧a } \\
\text { (PA) }\end{array}$ & 52 & $\mathbf{F}$ & $\begin{array}{c}\text { Icterícia, colúria, } \\
\text { hepatomegalia, dor em HD, } \\
\text { febre e perda ponderal; } \\
\text { Múltiplos cistos hepáticos } \\
\text { (RMA); } \\
\text { Sorologia Positiva. }\end{array}$ & Figado & - & Não & $\begin{array}{l}\text { Albendazol (600 } \\
\text { mg/dia); } \\
\text { Melhora do quadro } \\
\text { clinico, com } \\
\text { regressáo das lesóes } \\
\text { cisticas estando } \\
\text { atualmente } \\
\text { assintomática. }\end{array}$ & 26 \\
\hline 39 & $\begin{array}{l}\text { Capitĩo Poşo } \\
\text { (PA) }\end{array}$ & 36 & M & $\begin{array}{l}\text { Dor em HD com irradiaģão } \\
\text { para epigástrio, losse, } \\
\text { hepatomegalia e perda } \\
\text { penderal; } \\
\text { Lesōes cisticas em figado, sem } \\
\text { calcificaçōes (TCA); } \\
\text { Sorologia Positiva. }\end{array}$ & Figado & - & Nāo & $\begin{array}{c}\text { Albendzzol } \\
\text { (400mg/dia) } \\
\text { Desconhecida }\end{array}$ & $\mathrm{PT}$ \\
\hline 40 & $\begin{array}{c}\text { Barcarena } \\
\text { (PA) }\end{array}$ & 53 & $\mathbf{F}$ & $\begin{array}{l}\text { Assintomática; } \\
\text { Cistos hepáticos sem } \\
\text { calcificaçōes; } \\
\text { Sorologla Positiva. }\end{array}$ & Figado & - & Não & $\begin{array}{c}\text { Albendazol (800 } \\
\text { mg/dia) }\end{array}$ & PT \\
\hline
\end{tabular}

PA = Estado do Pará, Ev. = Echinococcus vogeli, AP = Estado do Amapá, E.sp. = Echinococcus espécies, $\mathbf{M}=$ masculino, F = feminino, Ref = Referência bibliográica, $\mathrm{HD}=$ Hipocôndrio direito, PT = presente trabalho, - = Informaçãa nã̃o obtida, TCA = Tomografia computadorizada abdominal, RMA = Ressonância magnética abdominal, USA = Ultra-sonografia abdominal.

para a inclusão nesta revisão/atualização. A Figura 6 demonstra a distribuição da casuística na área geográfica estudada. Sobressai-se a casuística da Ilha de Marajó (municípios de Anajás, Ponta de Pedras, Muaná, Breves, Afuá, e Curralinho). Só foram incluídos como critério de prova terapêutica os casos acompanhados com 0 controle de imagens pré e pós-tratamento com albendazol (Figura 7).

Não foram evidenciadas características compatíveis com Echinococcus granulosus ou E. oligarthrus ${ }^{21}$ entre os espécimes examinados ou reavaliados na presente atualização.

Dentre 17 pacientes com diagnóstico clínicoepidemiológico compatível com equinococose, examinados sorologicamente por immunoblot, $15(88,2 \%)$ mostram-se positivos.

\section{DISCUSSÃ0}

São notórias as dificuldades de enfrentamento da equinococose para a Amazônia brasileira. A principio pelo diminuto número de casos diagnosticados corretamente e divulgados. Freqüentemente é lembrada tardiamente como hipótese diagnóstica. De outra parte, emerge a dificuldade decorrente da sua base epidemiológica enraizada em situações de ordem sócioeconômica e cultural na relação do homem com 0 seu ambiente.

A revisão ora apresentada amplia para 40 os casos compatíveis com equinococose policística na Amazônia oriental brasileira e para 39 os casos procedentes do Estado do Pará. Considerando que a mais recente revisão mundial sobre equinococose neotropical realizada por D'Alessandro et $\mathrm{al}^{6}$, refere 72 casos da doença, com a presente atualização, elevar-se-ia essa casuística para, no mínimo, 106 casos (descontados os seis da Amazônia oriental já incluídos em ambas as casuísticas). Reconhece-se, todavia, que há muita subnotificação dos casos amazônicos e que vários grupos de pesquisadores estão publicando novos casos com maior frequiência, 0 que tende a tornar obsoleto 0 número de casos inicialmente apresentado. Um aspecto demográfico de avaliação interessante é a não predileção por sexo nos pacientes acometidos. Tal fato,concordando com outras casuísticas brasileiras ${ }^{13}$, clínica e etiologicamente bem documentadas, sugere que a transmissão doméstica e peridomiciliar é fundamental na manutenção dessa endemia neotropical.

No âmbito da distribuição geográfica da procedência da Amazônia oriental, chama a atenção, a grande concentração junto à Ilha de Marajó ( Figura 6). É possível que a prática da 


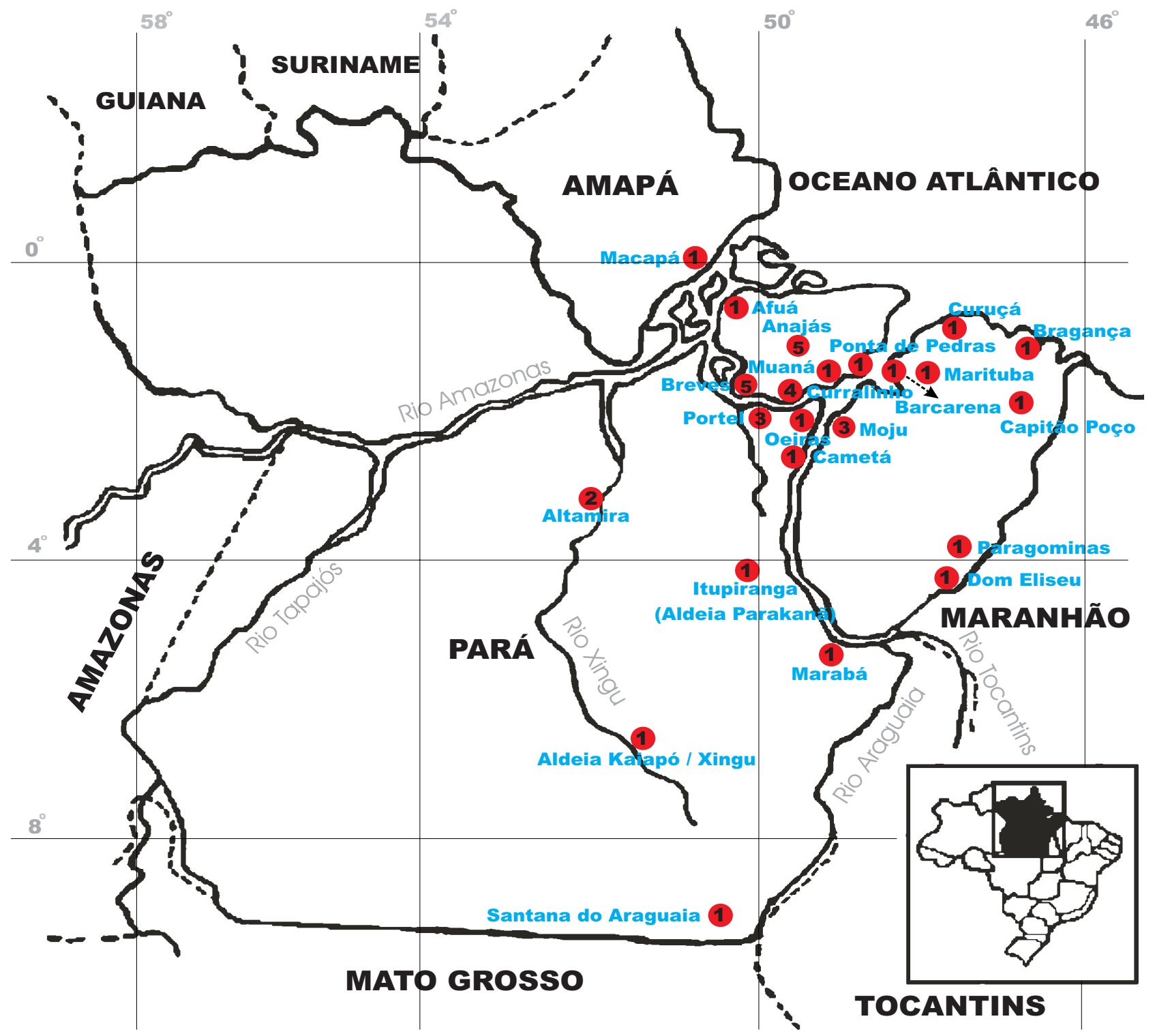

Localidade (número de casos)

Figura 6 - Distribuição geográfica dos casos de equinococose, com procedências definidas na Amazônia oriental brasileira.
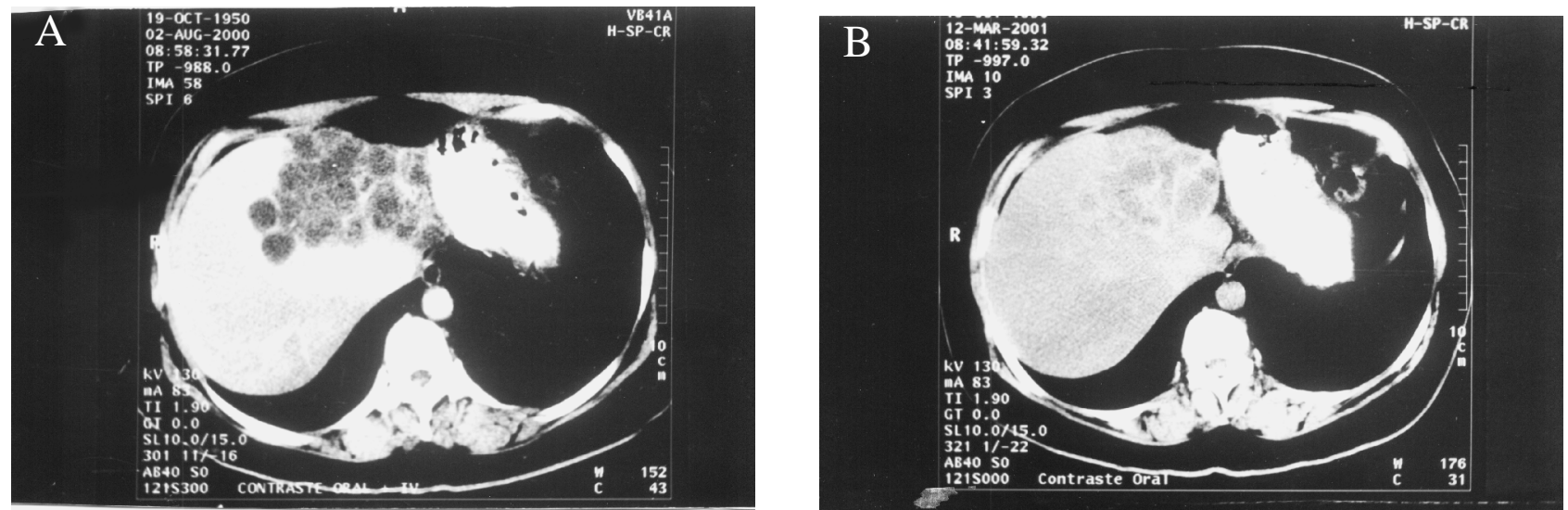

Figura 7 - Tomografia computadorizada mostrando lesões císticas hepáticas iniciais (A) e a regressão das mesmas (B) após tratamento clinico com albendazol na dose de $10 \mathrm{mg} / \mathrm{kg} /$ dia (prova terapêutica). 
caça de subsistência, ainda bastante presente naquela região, possa estar relacionada com a maior introdução do agente no ciclo doméstico da infecção. Neste particular, é freqüente o relato dos moradores sobre fígados de caça apresentando caroços, os quais são servidos como alimentos aos cães domésticos.

Existem diversas dificuldades em aferir uma real ou aproximada incidência da equinococose policística na Amazônia. 0 fato de constituir doença de longa evolução e com pouca ou nenhuma sintomatologia nos anos iniciais, a carência de métodos laboratoriais definidos e difundidos visando o diagnóstico etiológico, além do ainda insuficiente interesse da classe médica regional referente ao tema, constituem exemplos dessas dificuldades.

A constatação, concordando com a vasta literatura sobre 0 assunto 1469101315172324283031 , de que nesta casuística mais de $80 \%$ dos casos apresentavam lesões no fígado justifica a importância dessa parasitose no diagnóstico diferencial das hepatopatias regionais. Há coerência, por outro lado, na ausência de E. oligarthrus associado aos casos desta atualização, uma vez que só existe, na literatura médico-científica, o registro de lesões policísticas extra-hepáticas associadas a esse Echinococcus ${ }^{57}$. Fator adicional de singular importância na abordagem do problema é a atenção ao diagnóstico diferencial da equinococose policística, evitando dessa forma procedimentos retardados ou inadequados de diagnóstico, com graves consequiências para o doente. Por razões diversas, 0 diagnóstico diferencial com 0 hepatocarcinoma assume importância fundamental na região sob estudo. Cerca de 15\% da presente casuística foram encaminhados ao hospital de referência em oncologia, antes do diagnóstico definitivo. Contribui para a necessidade do acurado diagnóstico diferencial a importância da associação do vírus da hepatite B em hepatocarcinomas de pacientes procedentes da área silvestre amazônica.

Quando foi possível a avaliação histológica sobressaíramse, nas lesões hepáticas 0 aspecto "cerebróide" da camada laminar, lesão típica da equinococose por E. vogeli ${ }^{5613}$ (Figura 5). Considerando-se a importância do achado do acúleo do parasita para confirmar a etiologia, foi de grande valia para este estudo o concurso da microscopia óptica de polarização. Por meio desse artifício foi possível otimizar o encontro de acúleos inteiros ou fragmentados (Figuras 2 e 4). Com efeito, todos os casos com confirmação etiológica/parasitológica mostraram características de E. vogeli, incluindo alguns com revisão de equivocados ou insuficientes diagnósticos prévios. Em verdade, não é de nosso conhecimento a constatação parasitológica, na Amazônia brasileira, de casos de equinococose policística com outra etiologia. Reveste-se de interesse aos médicos, a coleta para exame microscópico de materiais eventualmente drenados de lesões suspeitas ou decorrentes de vômica, oriunda de cistos pulmonares ou de fístula hepato-pulmonar. Nesses materiais pode-se identificar acúleos e protoescóceles que indiquem 0 gênero e a espécie do Echinococcus.

A sorologia tem sido utilizada sob metodologias diversas no diagnóstico da equinococose. Testes de inibição da hemaglutinação, enzimaimunoensaio, immunoblot e outros têm sido aplicados com especificidades, sensibilidades e valores preditivos variados 25611202226 . 0 método de immunoblot utilizado neste estudo, em 17 casos, mostrou sensibilidade de 88,2\%. Reconhece-se a necessidade de validar comparativamente os diversos métodos sorológicos no âmbito da casuística brasileira pelos diversos Echinococcus prevalentes. É sabido sobre os fatores que podem influir no diagnóstico sorológico tais como a localização e crescimento dos cistos, a resposta imune do hospedeiro, a espécie ou cepa do parasito além, obviamente, da qualidade dos antígenos e demais reagentes.

Os profissionais da área de imagenologia têm prestado relevante serviço ao diagnóstico, uma vez alertados e após consolidarem experiência no padrão de imagem da equinococose policística. Ratifica-se que a presença de calcificações nas lesões císticas deve merecer devida atenção enquanto importante valor preditivo para equinococose ${ }^{13}$. Decorrente da estratégica condição exercida pela imagenologia alguns comentários merecem lembrança na rotina do diagnóstico: 0 RX continua como excelente abordagem preliminar, pois detecta melhor as calcificações, além de poder avaliar diversos órgãos com eventuais infecções associadas, especialmente o pulmão; a ultra-sonografia constituise em abordagem mais acessível para os pacientes, incluindo a possibilidade de eventuais levantamentos da doença nas populações rurais; a tomografia axial computadorizada, embora menos acessível e mais dispendiosa, agrega vantagens relativas à sua alta sensibilidade, incluindo a detecção de calcificações e a possibilidade de avaliar os diversos órgãos (Figura 7). A ressonância magnética merece abordagem especial para definir a sua utilidade, embora haja a referência de sua importância no acompanhamento das complicações da equinococose.

Sobre a ecoepidemiologia da doença para a Amazônia oriental e em apoio aos resultados ora apresentados, relatos preliminares referentes a duas áreas estudadas ( Ilha de Marajó e Área Indígena Parakanã) por Soares et al ${ }^{29}$ têm ratificado as observações de $D^{\prime}$ Alessandro et $\mathrm{l}^{8} \mathrm{e}$ Meneghelli etal ${ }^{14}$, referentes, principalmente, às presenças da infecção por E. vogeli em Agouti paca (paca) e E. oligarthrus em Dasyprocta aguti ( cutia). Como contribuição original, entretanto, tem-se o registro de E. vogeli em Dasypus novemcinctus ( tatu), na Ilha de Marajó29. Por outro lado, Silva Junior e Soares, 1999, registraram a presença do Speothos venaticus (cachorro-do-mato-vinagre), possível hospedeiro definitivo do E. vogeli, na Ilha de Marajón ${ }^{27}$.

A partir do reconhecimento da importância e das implicações do manejo da equinococose para a região tropical, acredita-se que deverá ocorrer uma implementação do diagnóstico precoce, tratamento adequado e de um melhor registro da doença.

\section{AGRADECIMENTOS}

A todos os servidores lotados na Seção de Hepatologia do Instituto Evandro Chagas, que pelas suas atividades tornaram possível o presente trabalho. Agradecimento especial aos técnicos Bernardo Farias da Conceição e Domingos Macedo de Souza, pela dedicação ao trabalho de campo. 


\section{REFERÊNCIAS BIBLIOGRÁFICAS}

1. Andrade HS, Rocha SMP. Hidatidose Hepática ( apresentação de um caso). Trabalho de conclusão de curso, medicina, Universidade Federal do Pará, Belém, PA, 1987.

2. Ayadi A, Dutoit E, Sendid B, Camus D. Specific diagnostic antigens of Echinococcus granulosus detected by westernblot. Parasite 2: 119-123, 1995.

3. Cruz ERM, Pinho CN, Silva CA, Alves MM, Cruz MM, Soares MCP. Hidatidose por E. vogeli - Apresentação de um novo caso do Estado do Pará. In: Resumos do XXIII Congresso Brasileiro de Patologia, Salvador, BA, 2001.

4. D'Alessandro A. Hidatidose Policística (E. vogeli e E. Oligarthrus). In: Veronesi R, Focaccia R (eds) Tratado de Infectologia, Atheneu, São Paulo, p.1413-1420, 1997.

5. D'Alessandro A. Polycystic echinococcosis in tropical America: Echinococcus vogeli and E. oligarthrus. Acta Tropica 67:43-65, 1997.

6. D'Alessandro A, Moares MAP, Raick AN. Polycystic Hydatid Disease in Brazil. Report of five new human cases and short review of other published observations. Revista da Sociedade Brasileira de Medicina Tropical 29:219-228, 1996.

7. D'Alessandro A, Ramirez LE, Chapadeiro E. Second recorded case of human infection by Echinococcus oligarthrus. American Journal Tropical Medicine Hygiene 52: 29-33, 1995.

8. D'Alessandro A, Rausch RL, Morales GA, Collet S, Angel D. Echinococcus infections in Colombian Animals. The American Journal of Tropical Medicine and Hygiene 30: 1263-1276, 1981.

9. Fonseca Jr OG, Costa AP. Hidatidose: comparação sobre 16 (dezesseis) casos para determinação microrregional de focos no Estado do Pará. Trabalho de conclusão de curso, medicina, Universidade Federal do Pará, Belém, PA, 1995.

10. Freitas AF, Sena CEB. Reavaliação Histológica e Parasitológica em Hidatidose-Equinococose no Hospital Universitário João de Barros Barreto (HUJBB). Trabalho de conclusão de curso, medicina, Universidade Federal do Pará, Belém, PA, 1999.

11. Gottstein B, D'Alessandro A, Rausch RL. Immunodiagnosis of polycystic hydatid disease/polycystic echinococosis due to Echinococcus vogeli. The American Journal of Tropical Medicine and Hygiene 53: 558-563, 1995.

12. Honório MG, Nascimento AP, Correia Lima FG, Eulálio KD. Hidatidose Abdominal e Pélvica, Relato de um Caso. In: Resumos do XXXVIII Congresso da Sociedade Brasileira de Medicina Tropical, 35: Supl.I, p.242, 2002.

13. Meneghelli UG, Martinelli ALC, Velludo MASL, Bellucci AD, Magro JE, Barbó MLP. Polycystic Hydatid Disease (Echinococcus vogeli). Clinical, laboratory and morphological findings in nine brazilian patients. Journal of Hepatology 14: 203-210, 1992.

14. Meneghelli UG, Martinelli ALC, Velludo MASL. Cistos de Echinococcus vogeli em Fígado de Paca (Cuniculus paca) Originária do Estado do Acre, Brasil. Revista da Sociedade Brasileira de Medicina Tropical 23: 153-155, 1990.

15. Miranda RA, Nascimento JRL, Soares MCP, Menezes RC. Hidatidose policística: Aspectos clínicos, epidemiológicos, sorológicos e de imagem. Tribo indígena Parakanã, Amazônia Oriental brasileira. Revista da Sociedade Brasileira de Medicina Tropical 31: Supl.I, p.54, 1998.

16. Nascimento Neto AA, Silva SPA, Pimentel IN. Equinococose Hepática: Caso autóctone no Estado do Pará. Trabalho de conclusão de curso, medicina, Universidade Federal do Pará, Belém, PA, 1983.
17. Nohmi N, Soherzinger CM, Brasileiro Filho G, Albuquerque R. Hidatidose: primeiro caso diagnosticado em Macapá, T. F. Amapá. Boletim do Hospital Escola São Camilo e São Luis 13/14:83-88, 1981.

18. Pacheco PRG, Komma MO, Pinto RNL, Souza LCS, Pereira LIA. Doença Hidática Policística. Relato de um caso procedente do Pará. Revista da Sociedade Brasileira de Medicina Tropical 19:67-68, 1986.

19. Passos DMM, Alves MP, Creuv O, Freitas G, Matushita JP, Marchiori E. Equinocose Alveolar Hepática (Descrição de um caso). Radiologia Brasileira 15:129-132, 1984

20. Pastore R, Vitali LH, Macedo VO, Prata A. Inquérito sorológico da infecção pelo Echinococcus sp. no município de Sena Madureira, AC. Revista da Sociedade Brasileira de Medicina Tropical 36: 473-477, 2003.

21. Rausch RL, Rausch VR, D'Alessandro A. Discrimination of the larval stages of Echinococcus oligarthrus ( Diesing, 1863) . American Journal Tropical Medicine Hygiene 27: 1195-1202, 1978.

22. Romani ELS. Determinação de antígenos relevantes da forma larvar do Echinococcus granulosus: Padronização e aplicação do "Immunoblot" no diagnóstico da hidatidose humana. Dissertação de Mestrado, Instituto Oswaldo Cruz, Rio de Janeiro, RJ, 1995.

23. Silva CAM, Alves MM, Almeida FB, Rodrigues-Silva R, Soares MCP. Novos Casos de Equinococose Policística no Brasil. In: Resumos do XVIII Congresso Brasileiro de Parasitologia, Rio de Janeiro p. 180, 2003.

24. Silva CAM, Alves MM, Conde SRS, Amaral IA, Teixeira LSC, Soares MCP. Hidatidose/ Equinococose na Amazônia Oriental Brasileira - Atualização da casuística. In Resumos do XXXVII Congresso da Sociedade Brasileira de Medicina Tropical. Revista da Sociedade Brasileira de Medicina Tropical 35: Supl.I, 243, 2002.

25. Silva CAM, Alves MM, Rocha BNS, Almeida FB, Couceiro MFG, Crescente JAB, Rodrigues-Silva R, Soares MCP. Equinococose Policística: Relato e Evolucãa de um Caso da Amazônia Oriental Brasileira. In: Resumos do VII Congresso Brasileiro de Clínica Médica, São Paulo p. OR075, 2003.

26. Silva CAM, Alves MM, Souza OSC, Brito EMF, Almeida FB, Soares MCP, Silva RR Avaliação sorológica no diagnóstico da equinococose policística. In: Resumos do XXXIV Congresso da Sociedade Brasileira de Medicina Tropical, Belém, Revista da Sociedade Brasileira de Medicina Tropical 36: Supl. I, p.190, 2003.

27. Silva Junior JS, Soares MCP. An Unexpected new record for the bush dog. Speothos venaticus Lund, 1842, in the brazilian Amazônia (Carnivora, Canidae). Publicações Avulsas do Instituto Pau Brasil de História Natural 2:7-11, 1999 .

28. Soares MCP, Amaral ISA. Polycystic echinococcosis by E. vogeli in the Amazon region. Journal of Hepatology 28:908, 1998.

29. Soares MCP, Cartágenes PRB, Alves MM, Silveira F, Amaral ISA. Registro de reservatórios silvestres para E. vogeli e E. Oligarthrus na Amazônia oriental brasileira. Gastroenterologia Endoscopia Digestiva (GED) 17( supl) : 153, 1998.

30. Soares MCP, Fonseca Jr OG, Alves MM, Cartágenes PRB, Amaral ISA, Freitas AF, Sena CEB, Crescente A, Bensabath G. Equinococose na Amazônia oriental brasileira. Atualização da casuística incluindo casos com revisão etiológica. In: Resumos do I Congresso Norte de Infectologia, Belém, PA. Revista Paraense de Medicina 14: 45, 2000.

31. Teixeira LSM, Albério CAA, Silva CA, Soares MCP. Hidatidose hepática Seguimento clínico de 12 meses. In: Resumos do VI Congresso Brasileiro de Clínica Médica e I Congresso Brasileiro de Acadêmicos em Clínica Médica, Curitiba p. 119, 2001. 